\title{
Structural and energetic analysis of cyclic peptide-gold nano-drug delivery system: a DFT study
}

\author{
B. Khoshbayan, A. Morsali, M. R. Bozorgmehr, S. A. Beyramabadi \\ Department of Chemistry, Mashhad Branch, Islamic Azad University, Mashhad, Iran \\ bahareh.khoshbayan@gmail.com, almorsali@yahoo.com,mr_bozorgmehr@yahoo.com, abeyramabadi@gmail.com
}

PACS 78.67.-n, 78.67.Ch

DOI 10.17586/2220-8054-2021-12-5-612-622

\begin{abstract}
By applying cyclooctaglycine model for cyclic peptide (CP) and cluster Au6 model for gold nanoparticles (GN), seven different configurations of cyclic peptide-gold nanoparticles (CPGN) with 5-fluorouracil (FU) were investigated. Binding energies, quantum molecular descriptors, and solvation energies in the aqueous solution and gas phase were studied at the density functional level of M06-2X/6-31g(d, p). Solvation energies indicate that the solubility of FU increases in CPGN/FU1-7. This subject is considered a key factor for drug transfer, so CPGNs can be used as an appropriate drug delivery system. The large negative values of calculated binding energies show the stability of CPGN/FU1-7 structures, and quantum molecular descriptors, such as electrophilicity $(\omega)$ and global hardness $(\eta)$ indicate that the reactivity of FU in CPGN/FU1-7 structures increases. AIM calculations for all structures also show that intermolecular hydrogen bonding and Au-drug interactions play an important role for this drug delivery system.
\end{abstract}

Keywords: 5-fluorouracil, AIM, drug delivery, DFT, cyclic peptide-gold nanosystem.

Received: 4 May 2021

Revised: 22 July 2021

\section{Introduction}

Drug delivery systems have recently been used as new structures for better drug performance. Various organic and inorganic drug delivery systems such as drug-polymer conjugates [1,2], carbon nanotubes [3,4], gold nanoparticles (NPs) [5], magnetic NPs [6,7], silica NPs [8] and other inorganic nanosystems [9-11] have been investigated due to their chemical stability and environmental compatibility as well as reduced toxicity in medical activities.

In recent studies, gold nanoparticles (GNs) have received significant attention because they have the ability to select and identify cancer cells compared to normal cells [12]. For this reason, GNs have been employed as nanocarriers of different anticancer drugs [5,13]. In addition to targeted drug delivery, they can perform a controlled release [14,15].

Cyclic peptides are polypeptide chains with a circular structure and have many uses in various contexts such as drug delivery, nanoscience, optical sensors, and electronic devices. The ring structure is formed by the end of one peptide to the other section with an amide coupling. The cyclic structure with different chain amino acids is a good pattern for encapsulating medicines [16-19]. These structures have been useful in many applications such as molecular transport systems [20,21], antibacterial agents [22,23] and other applications [24]. Peptides are less toxic than synthetic molecules and therefore do not accumulate in the tissue. Cyclic peptide-based drugs can cause less harm and usually show better biological activities than their linear counterparts due to their rigid configuration. These drug delivery systems have a variety of structural properties leading to their use as a target drug binder in medical applications [24-26]. Also, they have the ability to penetrate cells more than their linear counterparts and therefore, cellular uptake is significantly increased [27,28].

Over the past few years, the use of cyclic peptides in the transport of a wide range of therapeutic agents (including anticancer drugs, anti-HIV drugs, and essential phosphopeptides) has been investigated [26, 29, 30]. Recently, gold nanoparticles along with cyclic peptides (CPGNs) have been used as drug transporters for anticancer drugs [31].

Along with experimental methods, quantum mechanical methods are used to better understand drug delivery and biochemical systems [32-37]. In this study, density functional theory (DFT) was applied for exploring gold nanoparticles-cyclic peptide (CPGNs) with 5-fluorouracil (FU) as a drug delivery system. Although this drug (FU) was introduced more than thirty years ago, it is still one of the most widely used anti-cancer drugs for the treatment of many different malignancies alone or in combination with other drugs. FU has shown activity in breast, brain, gastrointestinal (especially colon cancer) and ovarian cancers [38-42]. 


\section{Computational details}

DFT calculations were performed at the density functional level of M06-2X with 6-31G(d, p) basis set by GAUSSIAN 09 [43]. For all structures, the optimization of the degrees of freedom in the gas phase and aqueous solution has been performed. Solvent effects were investigated using the polarized continuum model (PCM). Solvation energies $\left(\Delta E_{\text {solv }}\right)$ were calculated by the following equation:

$$
\Delta E_{\text {solv }}=E_{\text {sol }}-E_{\text {gas }}
$$

where $E_{\text {sol }}$ and $E_{\text {gas }}$ represent the total energy for the solution and gas phases. Calculations for cyclic peptide (CP), gold nanoparticles (GN), and 5-fluorouracil (FU) as well as seven CPGN/FU 1-7 configurations were examined.

Quantum molecular descriptors are used to study the stability of different structures. The global hardness $(\eta)$ indicates the resistance to changing electronic structure and it is determined by the following equation:

$$
\eta=\frac{I-A}{2}
$$

where $A=-E_{L U M O}$ and $I=-E_{H O M O}$ are the electron affinity and the ionization potential, in the respective order. The term HOMO-LUMO refers to the highest occupied molecular orbital-lowest unoccupied molecular orbital energy. The electrophilicity index $(\omega)[44]$ is obtained by the following equation:

$$
\omega=\frac{(I+A)^{2}}{8 \eta} .
$$

We used the quantum theory of atom in molecules (QTAIMs) to study the nature of created bonds. QTAIM is based on electron density analysis, $\rho(r)$. AIM calculations were performed using AIMAII software [45]. Different values of electron density such as potential energy density $\left(V_{b}\right)$, kinetic energy density $\left(G_{b}\right)$, total energy density $\left(H_{b}\right)$ and Laplacian of electron density $\left(\nabla^{2} \rho\right)$ at the bond critical point (BCP) were examined for specifying the nature of the bond in different structures.

\section{Results and discussion}

Cyclooctaglycine [46] and Au6 cluster [47] were used for cyclic peptide (CP) and gold nanoparticles (GN) modelling, respectively. The different configurations of small Au clusters with 3 to 20 atoms are well known due to numerous experimental and theoretical studies [48-53]. The interaction of gold nanoparticles - cyclic peptide (CPGN) with 5-fluorouracil (FU) was investigated from seven different directions. 5-fluorouracil (Fig. 1) has functional groups such as (F, NH and $\mathrm{CO})$. The various configurations in which 5-fluorouracil approaches CPGN through its functional groups are called CPGN/FU1-7. The optimized structures of (FU), (GN), (CP) and (CPGN/FU1-7) in the aqueous solution are shown in Fig. 2.

The binding energies $(\Delta E)$ for different structures were calculated using the following equation:

$$
\Delta E=E_{C P G N / F U 1-7}-\left(E_{C P}+E_{G N}+E_{F U}\right) .
$$

Table 1 shows the binding energies in the gas phase $\left(-278.341 \mathrm{~kJ} \cdot \mathrm{mol}^{-1}\right.$ on average) and aqueous solution $\left(-234.219 \mathrm{~kJ} \cdot \mathrm{mol}^{-1}\right.$ on average $)$ at the M06-2X level. The amounts of these energies in the aqueous solution are lower than in the gas phase; however, their large negative values in both phases indicate the exothermic and optimal performance of CPGN with FU drug. Binding energies depend on the orientation of the drug relative to CPGN. Of the seven species studied in the solution phase, CPGN/FU2 is the most stable configuration. In this configuration, the FU drug is parallel to $\mathrm{CPGN}$, and the $\mathrm{NH}$ and $\mathrm{OH}$ functional groups of $\mathrm{CP}$ interact with the FU drug (Fig. 2). The values of binding energies show the stability of the structures in which FU drug interacts simultaneously with CP and GN. In species where FU interacts only with GN, such as CPGN/FU7, the least stability is observed.

In this research, we evaluated the solvation energy $\left(\Delta E_{\text {solv }}\right)$ for all species (Table 1). The obtained values are negative, indicating the spontaneous solvation, which is very important in drug delivery systems and it is an essential factor for an effective anti-cancer drug. In order to determine the share of each of the two carriers (CP and GN) in the binding and solvation energies, the FU drug was optimized separately near CP and GN (CP/FU and GN/FU in Fig. 1 and Table 1). The solvation of the drug and GN increases in the presence of CP. An important feature of the cyclic peptide is its $\mathrm{NH}$ and $\mathrm{CO}$ functional groups, which form a hydrogen bond between the FU drug, solvent molecules, and CP. In fact, CP and GN complement each other. GN increases binding energy, and CP increases solvation, and the two become together as a carrier, with better properties.

The stability of various configurations was also investigated using quantum molecular descriptors such as electrophilicity index $(\omega)$ and global hardness $(\eta)$ using the HOMO-LUMO energy gap $\left(E_{g}\right)$. Table 1 shows quantum molecular descriptors such as global hardness $(\eta)$ and electrophilicity strength $(\omega)$ for FU, CP, GN, CP/FU, GN/FU and CPGN/FU1-7 in aqueous solution and gas phases. According to Table 1, in the solution phase, the FU drug and 

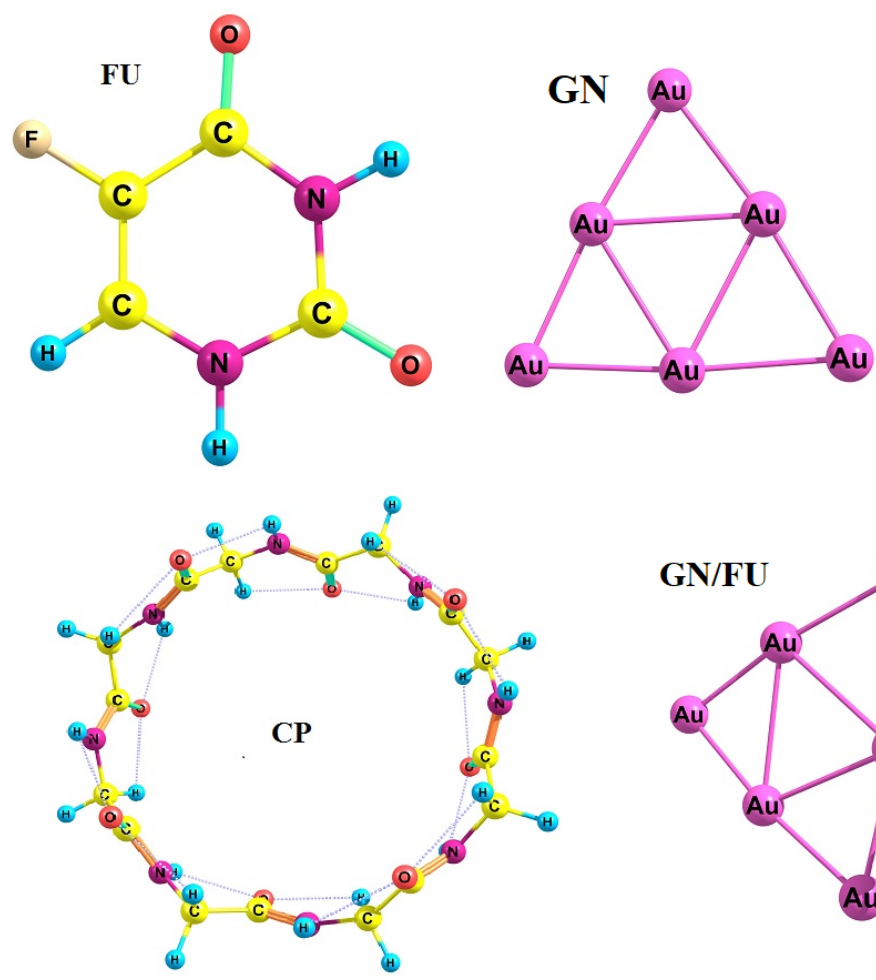

GN/FU

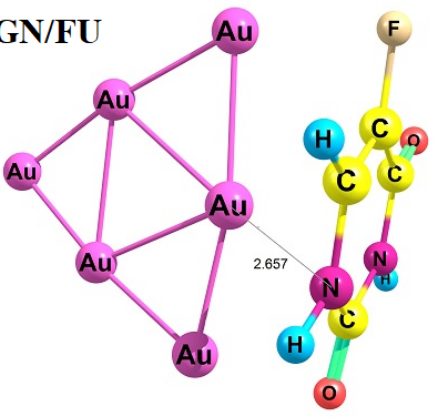

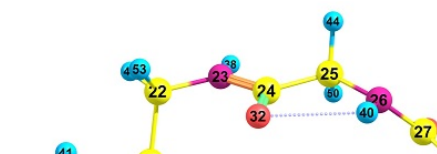

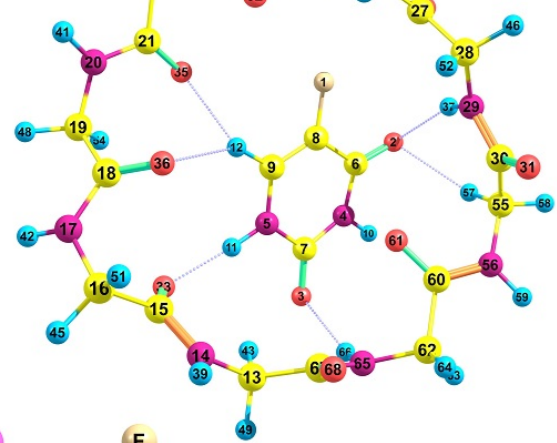

$\mathbf{C P} / \mathbf{F U}$

FIG. 1. Optimized structures of FU, GN, CP/FU, CP and GN/FU

CP have high hardness values of 3.97 and $4.95 \mathrm{eV}$ and have high energy gap values of 7.95 and $9.89 \mathrm{eV}$, and they are considered highly stable species. However, GN is less stable than FU and CP due to hardness value of $2.69 \mathrm{eV}$ and gap energy of $5.38 \mathrm{eV}$. For CPGN/FU1-7 configurations, the obtained values of $E_{g}$ and $\eta$ are less than CP and FU drug, indicating a decrease in the stability of FU drug in the presence of gold nanoparticles and cyclic peptide. According to Table 1, the values of $\omega$ in CPGN/FU1-7 structures are higher than FU drug in both phases, which indicates the role of 5-fluorouracil drug as an electron acceptor in the drug delivery system and therefore, the charge is flowing from the carrier to the drug.

Now, a comprehensive review of intermolecular interactions is done using AIM analysis about the nature and strength of interactions through analysis of bond critical points BCP. The electron density $\rho(r)$ and its Laplacian $\nabla^{2} \rho$ are related to the strength and nature of a bond, respectively. The larger the value of $\rho(r)$, the stronger the bond. Also $\nabla^{2} \rho$ and $H_{b}$ show more information about the nature of the interactions. When $\left(\nabla^{2} \rho<0, H_{b}<0\right),\left(\nabla^{2} \rho>0\right.$, $\left.H_{b}<0\right)$ and $\left(\nabla^{2} \rho>0, H_{b}>0\right)$, the reactions will be strong, medium and weak, respectively. For parameter of $-G_{b} / V_{b}$. If $-G_{b} / V_{b}>1,0.5<-G_{b} / V_{b}<1$ and $-G_{b} / V_{b}<0.5$, the bonds will be noncovalent, partially covalent and covalent, respectively [54].

The structure of CPGN/FU2 as the most stable configuration and the structure of CPGN/FU7 as the most unstable configuration in details have been reported by AIM analysis. The values obtained from $\rho(r), \nabla^{2} \rho, H_{b}, G_{b}, V_{b}$ and $-G_{b} / V_{b}$ at the bond critical points for the CPGN/FU2 structure are shown in Table 2. The molecular diagram for the most stable state in the solution phase is also shown in Fig. 3. By using $E_{H B}=V_{b} / 2$, hydrogen bond energies can be calculated [55]. The Au-Au interactions in GN, according to Table 2 with the values of $\nabla^{2} \rho>0, H_{b}<0$ and $0.5<-G_{b} / V_{b}<1$ represent partially covalent bonds, which is the same for other structures. Negative values of $H_{b}$ indicate covalent bonds (even though closed shell interactions are also involved). Positive values of $\nabla^{2} \rho$ indicate the electrostatic interactions of the $\mathrm{Au}-\mathrm{Au}$ interaction [56].

In the CPGN/FU1-7 configurations, we encounter two groups of important interactions, the first of which is between $\mathrm{GN}$ and $\mathrm{CP}$ or GN and the FU drug. These interactions are in the form of $\mathrm{Au}-\mathrm{A}$ in which $\mathrm{A}$ includes $\mathrm{H}, \mathrm{O}$, $\mathrm{F}, \mathrm{C}$ and $\mathrm{N}$ atoms. The higher the $\mathrm{Au}-\mathrm{A}$ interactions and the higher the values of $\rho(r)$ and $\nabla^{2} \rho$, the more stable the configuration.

The CPGN/FU2 configuration has $20 \mathrm{Au}-\mathrm{A}$ interactions with values of $\rho_{a v}=0.0113$ and $\nabla^{2} \rho_{a v}=0.0392$ (as average). 3 interactions of Au-A with $\nabla^{2} \rho>0, H_{b}<0$ and $0.5<-G_{b} / V_{b}<1$ are partially covalent interactions (medium interactions) and $17 \mathrm{Au}-\mathrm{A}$ interactions, according to Table 2, are components of weak interactions with 


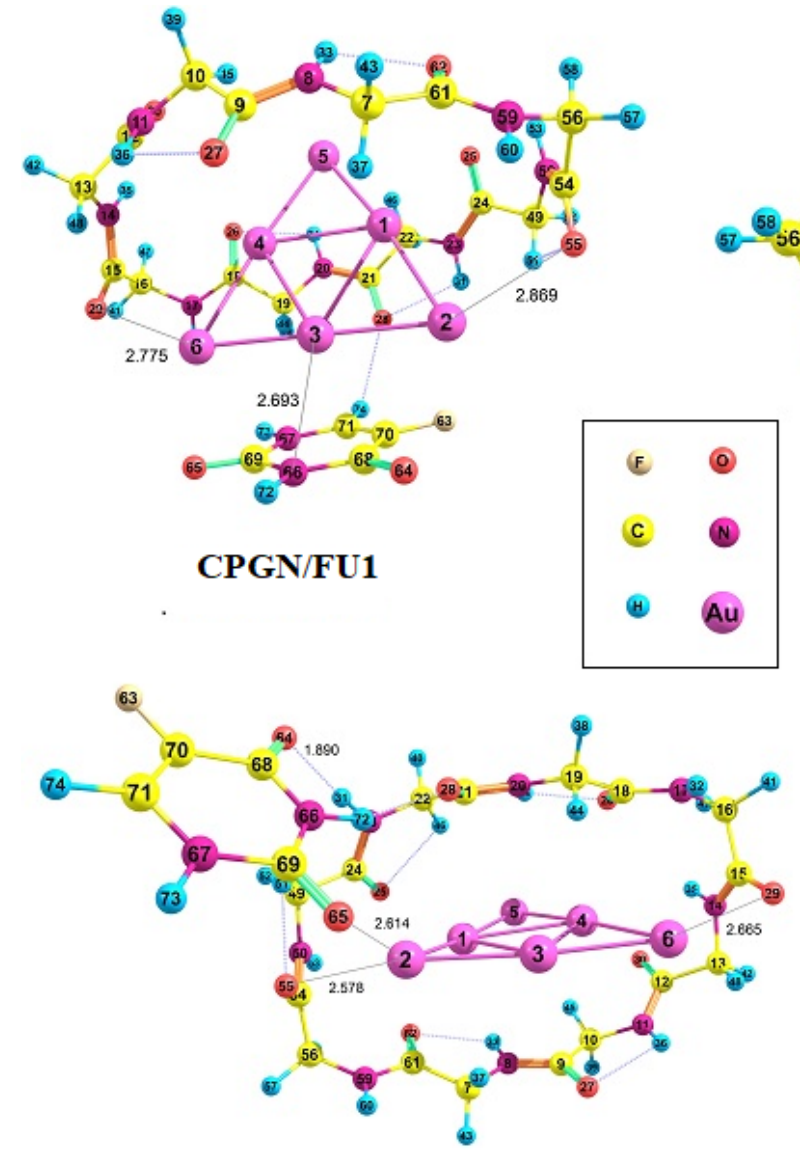

CPGN/FU3

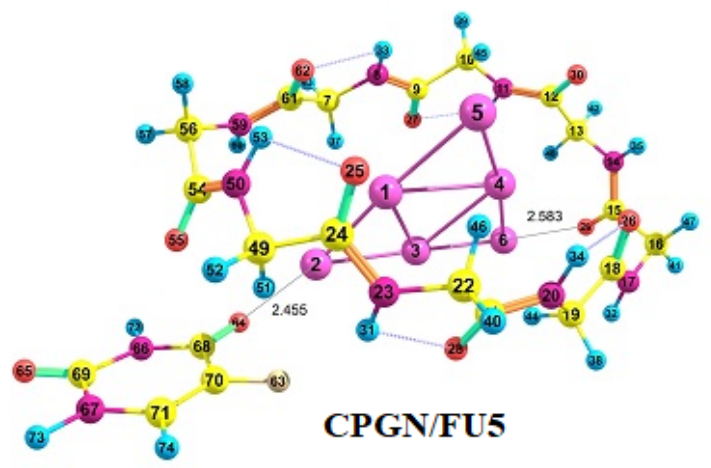

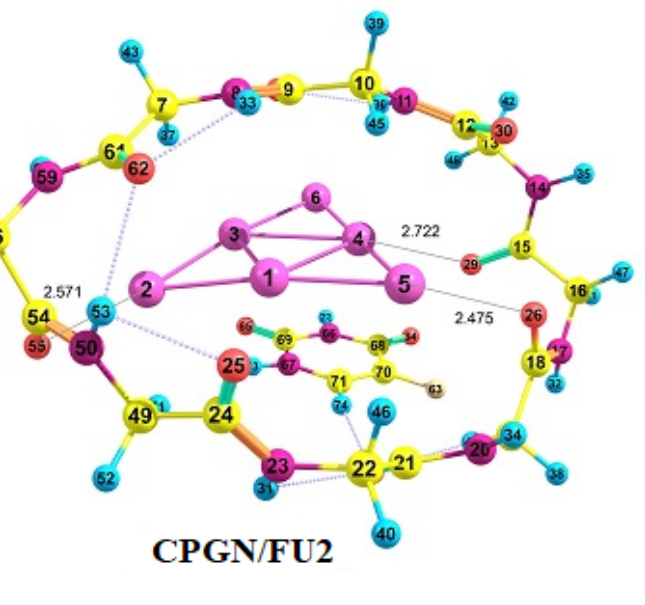

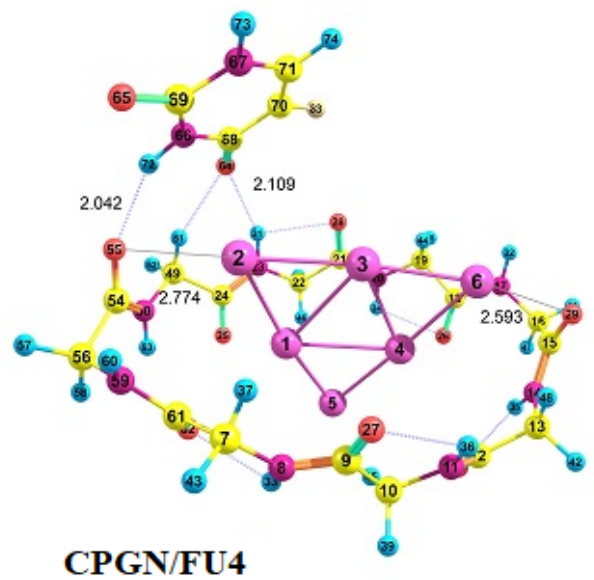

CPGN/FU4

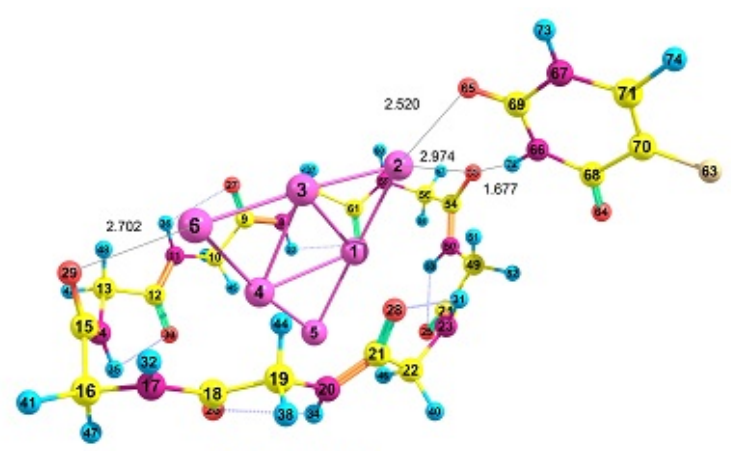

CPGN/FU6

CPGN/FU7

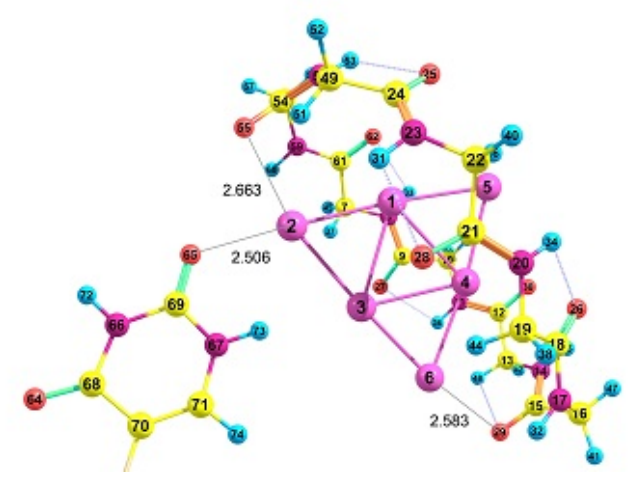

FIG. 2. Optimized structures of CPGN/FU1-7 

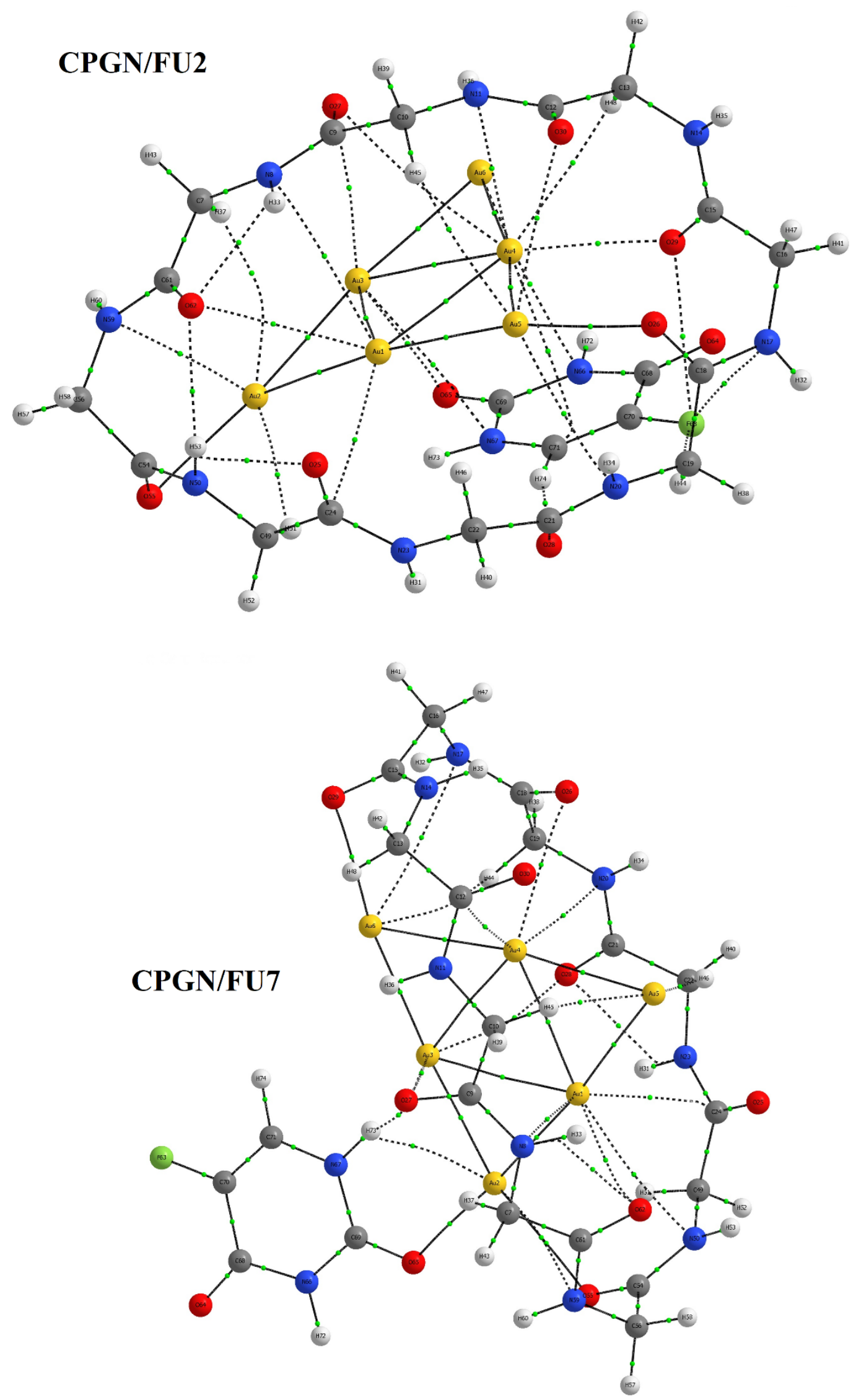

FIG. 3. Molecular graphs of CPGN/FU2 and CPGN/FU7. Small green spheres and lines related to the bond critical points (BCP) and the bond paths, respectively 
TABLE 1. Quantum molecular descriptors $(\mathrm{eV})$, binding $(\Delta E)$ and solvation $\left(\Delta E_{\text {solv }}\right)$ energies $\left(\mathrm{kJ} \mathrm{mol}^{-1}\right)$ for all structures

\begin{tabular}{|c|c|c|c|c|c|c|c|}
\hline Species & $E_{L U M O}$ & $E_{H O M O}$ & $E_{g}$ & $\eta$ & $\omega$ & $\Delta E$ & $\Delta E_{\text {solv }}$ \\
\hline \multicolumn{8}{|c|}{$\mathrm{H}_{2} \mathrm{O}$} \\
\hline $\mathbf{F U}$ & -0.119 & -8.071 & 7.952 & 3.976 & 2.109 & - & -40.9 \\
\hline $\mathbf{C P}$ & 1.395 & -8.499 & 9.894 & 4.947 & 1.275 & - & -92.2 \\
\hline GN & -1.556 & -6.933 & 5.377 & 2.688 & 3.350 & - & -24.4 \\
\hline CP/FU & -0.356 & -8.255 & 7.899 & 3.949 & 2.347 & -119.8 & -122.8 \\
\hline GN/FU & -1.570 & -6.862 & 5.291 & 2.645 & 3.360 & -42.4 & -47.5 \\
\hline CPGN/FU1 & -1.531 & -6.624 & 5.092 & 2.546 & 3.265 & -239.4 & -143.1 \\
\hline CPGN/FU2 & -1.420 & -6.486 & 5.065 & 2.532 & 3.085 & -243.6 & -151.2 \\
\hline CPGN/FU3 & -1.554 & -6.504 & 4.950 & 2.475 & 3.279 & -235.0 & -109.7 \\
\hline CPGN/FU4 & -1.643 & -6.639 & 4.995 & 2.497 & 3.433 & -231.8 & -90.2 \\
\hline CPGN/FU5 & -1.544 & -6.608 & 5.064 & 2.532 & 3.281 & -237.1 & -101.0 \\
\hline CPGN/FU6 & -1.544 & -6.654 & 5.112 & 2.556 & 3.285 & -217.5 & -73.7 \\
\hline CPGN/FU7 & -1.595 & -6.623 & 5.027 & 2.513 & 3.358 & -119.8 & -40.9 \\
\hline \multicolumn{8}{|c|}{ gas } \\
\hline $\mathbf{F U}$ & -0.256 & -8.246 & 7.990 & 3.995 & 2.262 & - & - \\
\hline $\mathbf{C P}$ & 1.340 & -8.575 & 9.916 & 4.958 & 1.319 & - & - \\
\hline GN & -2.317 & -7.562 & 5.244 & 2.622 & 4.653 & - & - \\
\hline CP/FU & -0.191 & -8.084 & 7.892 & 3.946 & 2.169 & -208.8 & - \\
\hline GN/FU & -2.268 & -7.416 & 5.147 & 2.573 & 4.554 & -60.2 & - \\
\hline CPGN/FU1 & -1.750 & -6.571 & 4.821 & 2.410 & 3.591 & -299.2 & - \\
\hline CPGN/FU2 & -1.436 & -6.454 & 5.017 & 2.508 & 3.102 & -301.4 & - \\
\hline CPGN/FU3 & -1.779 & -6.753 & 4.973 & 2.486 & 3.660 & -282.9 & - \\
\hline CPGN/FU4 & -1.633 & -6.595 & 4.961 & 2.480 & 3.411 & -253.8 & - \\
\hline CPGN/FU5 & -1.622 & -6.494 & 4.872 & 2.436 & 3.381 & -293.6 & - \\
\hline CPGN/FU6 & -1.672 & -6.666 & 4.993 & 2.496 & 3.481 & -250.0 & - \\
\hline CPGN/FU7 & -1.882 & -6.638 & 4.755 & 2.377 & 3.816 & -208.8 & - \\
\hline
\end{tabular}

$\left(\nabla^{2} \rho>0, H_{b}>0\right.$ and $\left.-G_{b} / V_{b}>1\right)$. The second group is the interaction between FU drug and $\mathrm{CP}$ via hydrogen bonding. In the CPGN/FU2 configuration, the H44 $\cdots$ F63 and $\mathrm{H} 74 \cdots \mathrm{O} 28$ bonds with $\nabla^{2} \rho>0, H_{b}>0$ and $-G_{b} / V_{b}>1$, are related to weak hydrogen bonds.

CPGN/FU7 is the most unstable structure in which there is no interaction between the drug and the cyclic peptide. For this structure, the molecular diagram and the values of $\rho(r), \nabla^{2} \rho, H_{b}, G_{b}, V_{b}$ and $-G_{b} / V_{b}$ in the solution phase are presented in Fig. 3 and Table 3. In this configuration, the drug approaches GN from the $\mathrm{NH}$ and $\mathrm{OH}$ groups. Considering Table 3, this configuration has $19 \mathrm{Au}-\mathrm{A}$ interactions with the values of $\rho_{a v}=0.0120$ and $\nabla^{2} \rho_{a v}=0.0419$ (on average). $3 \mathrm{Au}-\mathrm{A}$ interactions are medium and $16 \mathrm{Au}-\mathrm{A}$ interactions are weak. In this structure, there is no hydrogen bond between $\mathrm{CP}$ and FU. 
TABLE 2. Topological parameters in a.u. for CPGN/FU2

\begin{tabular}{|c|c|c|c|c|c|c|}
\hline Atoms & $\rho(r)$ & $\nabla^{2} \rho$ & $V_{b}$ & $G_{b}$ & $-G_{b} / V_{b}$ & $H_{b}$ \\
\hline \multicolumn{7}{|c|}{$\mathrm{Au}-\mathrm{Au}$ interactions } \\
\hline Au1 - Au3 & 0.03196 & 0.08931 & -0.03225 & 0.02729 & 0.84613 & -0.00496 \\
\hline $\mathrm{Au} 1-\mathrm{Au} 2$ & 0.04150 & 0.09884 & -0.04457 & 0.03464 & 0.77717 & -0.00993 \\
\hline $\mathrm{Au} 1-\mathrm{Au} 4$ & 0.03460 & 0.09201 & -0.03541 & 0.02920 & 0.82480 & -0.00620 \\
\hline $\mathrm{Au} 2-\mathrm{Au} 3$ & 0.06320 & 0.12105 & -0.07302 & 0.05164 & 0.70721 & -0.02138 \\
\hline $\mathrm{Au} 3-\mathrm{Au} 4$ & 0.03318 & 0.08992 & -0.03376 & 0.02812 & 0.83288 & -0.0056 \\
\hline $\mathrm{Au} 1-\mathrm{Au} 5$ & 0.06291 & 0.11411 & -0.07120 & 0.04986 & 0.70033 & -0.02133 \\
\hline $\mathrm{Au} 4-\mathrm{Au} 5$ & 0.04371 & 0.10296 & -0.04806 & 0.03690 & 0.76777 & -0.01116 \\
\hline Au3 - Au6 & 0.04720 & 0.10709 & -0.05265 & 0.03971 & 0.75423 & -0.01294 \\
\hline Au4 - Au6 & 0.05339 & 0.11867 & -0.06184 & 0.04575 & 0.73984 & $-0.0160 s$ \\
\hline \multicolumn{7}{|c|}{$\mathrm{Au}-\mathrm{A}$ interactions } \\
\hline $\mathrm{N} 8-\mathrm{Au} 1$ & 0.00728 & 0.01999 & -0.00408 & 0.00454 & 1.11190 & 0.00045 \\
\hline $\mathrm{H} 37-\mathrm{Au} 2$ & 0.00640 & 0.01510 & -0.00252 & 0.00315 & 1.24703 & 0.00062 \\
\hline $\mathrm{O} 27-\mathrm{Au} 3$ & 0.00593 & 0.01884 & -0.00328 & 0.00399 & 1.21717 & 0.00071 \\
\hline $\mathrm{N} 11-\mathrm{Au} 4$ & 0.01155 & 0.03406 & -0.00747 & 0.00799 & 1.06970 & 0.00052 \\
\hline H45 - Au5 & 0.00707 & 0.02089 & -0.00321 & 0.00421 & 1.31329 & 0.00100 \\
\hline $\mathrm{O} 30-\mathrm{Au} 5$ & 0.00759 & 0.02432 & -0.00460 & 0.00534 & 1.16083 & 0.00074 \\
\hline $\mathrm{H} 48-\mathrm{Au} 4$ & 0.00763 & 0.02725 & -0.00406 & 0.00544 & 1.33751 & 0.00137 \\
\hline $\mathrm{H} 29-\mathrm{Au} 4$ & 0.02409 & 0.09375 & -0.02439 & 0.02391 & 0.98048 & -0.00047 \\
\hline N20 - Au5 & 0.01095 & 0.03403 & -0.00717 & 0.00783 & 1.09330 & 0.00066 \\
\hline $\mathrm{C} 24-\mathrm{Au} 1$ & 0.00642 & 0.02172 & -0.00311 & 0.00427 & 1.37107 & 0.00115 \\
\hline O26 - Au5 & 0.03851 & 0.16091 & -0.04559 & 0.04291 & 0.94111 & -0.00268 \\
\hline $\mathrm{O} 27-\mathrm{Au} 4$ & 0.00507 & 0.01423 & -0.00247 & 0.00301 & 1.21742 & 0.00053 \\
\hline $\mathrm{H} 51-\mathrm{Au} 2$ & 0.00763 & 0.02531 & -0.00390 & 0.00511 & 1.31029 & 0.00121 \\
\hline $\mathrm{O} 62-\mathrm{Au} 1$ & 0.00489 & 0.01497 & -0.00235 & 0.00305 & 1.29334 & 0.00069 \\
\hline $\mathrm{O} 55-\mathrm{Au} 2$ & 0.03218 & 0.12694 & -0.03593 & 0.03383 & 0.94153 & -0.00210 \\
\hline N59 - Au2 & 0.01010 & 0.02853 & -0.00619 & 0.00666 & 1.07570 & 0.00046 \\
\hline $\mathrm{C} 71-\mathrm{Au} 4$ & 0.00923 & 0.02823 & -0.00477 & 0.00591 & 1.23895 & 0.00114 \\
\hline $\mathrm{O} 65-\mathrm{Au} 3$ & 0.01033 & 0.03463 & -0.00730 & 0.00798 & 1.09235 & 0.00067 \\
\hline N66 - Au6 & 0.00593 & 0.01448 & -0.00284 & 0.00323 & 1.13593 & 0.00038 \\
\hline N67 - Au3 & 0.00848 & 0.02716 & -0.00518 & 0.00598 & 1.15559 & 0.00080 \\
\hline \multicolumn{7}{|c|}{ Intermolecular hydrogen bonds } \\
\hline $\mathrm{H} 44-\mathrm{F} 63$ & 0.01072 & 0.04407 & -0.00897 & 0.00999 & 1.11415 & 0.00102 \\
\hline $\mathrm{O} 28-\mathrm{H} 74$ & 0.01842 & 0.05928 & -0.01479 & 0.01476 & 1.00176 & -0.00002 \\
\hline
\end{tabular}


Table 3: Topological parameters in a.u. for CPGN/FU1, CPGN/FU3-7 and $\mathrm{GN} / \mathrm{H}_{2} \mathrm{O}$

\begin{tabular}{|c|c|c|c|c|c|c|}
\hline Atoms & $\rho(r)$ & $\nabla^{2} \rho$ & $V_{b}$ & $G_{b}$ & $-G_{b} / V_{b}$ & $H_{b}$ \\
\hline \multicolumn{7}{|c|}{$\mathrm{Au}-\mathrm{Au}$ interactions (CPGN/FU3-7) } \\
\hline $\mathrm{Au} 1-\mathrm{Au} 3$ & 0.03801 & 0.10021 & -0.04071 & 0.03288 & 0.80769 & -0.00782 \\
\hline $\mathrm{Au} 1-\mathrm{Au} 2$ & 0.05995 & 0.11259 & -0.06754 & 0.04784 & 0.70836 & -0.01969 \\
\hline $\mathrm{Au} 2-\mathrm{Au} 3$ & 0.05361 & 0.10714 & -0.05929 & 0.04304 & 0.72585 & -0.01625 \\
\hline $\mathrm{Au} 1$ - Au4 & 0.03207 & 0.08994 & -0.03267 & 0.02758 & 0.84407 & -0.00509 \\
\hline $\mathrm{Au} 3$ - Au4 & 0.03387 & 0.09317 & -0.03504 & 0.02916 & 0.83237 & -0.00587 \\
\hline $\mathrm{Au} 1-\mathrm{Au} 5$ & 0.04794 & 0.10850 & -0.05379 & 0.04045 & 0.75215 & -0.01333 \\
\hline $\mathrm{Au} 4$ - Au5 & 0.05377 & 0.11737 & -0.06196 & 0.04565 & 0.73679 & -0.01630 \\
\hline Au3 - Au6 & 0.05861 & 0.11364 & -0.06633 & 0.04737 & 0.71414 & -0.01896 \\
\hline Au4 - Au6 & 0.04927 & 0.11032 & -0.05550 & 0.04154 & 0.74844 & -0.01396 \\
\hline \multicolumn{7}{|c|}{ Au-A interactions (CPGN/FU3-7) } \\
\hline $\mathrm{N} 8-\mathrm{Au} 1$ & 0.00768 & 0.02278 & -0.00464 & 0.00516 & 1.11328 & 0.00052 \\
\hline $\mathrm{O} 27-\mathrm{Au} 3$ & 0.00403 & 0.01234 & -0.00188 & 0.00248 & 1.31709 & 0.00059 \\
\hline $\mathrm{H} 45-\mathrm{Au} 5$ & 0.00790 & 0.02284 & -0.00360 & 0.00465 & 1.29165 & 0.00105 \\
\hline $\mathrm{C} 12-\mathrm{Au} 4$ & 0.01024 & 0.03900 & -0.00654 & 0.00814 & 1.24472 & 0.00160 \\
\hline N17 - Au6 & 0.00693 & 0.01894 & -0.00377 & 0.00425 & 1.12678 & 0.00047 \\
\hline $\mathrm{O} 26-\mathrm{Au} 4$ & 0.01296 & 0.04391 & -0.01000 & 0.01049 & 1.04857 & 0.00048 \\
\hline H44 - Au6 & 0.00608 & 0.01571 & -0.00246 & 0.00319 & 1.29643 & 0.00073 \\
\hline $\mathrm{N} 20-\mathrm{Au} 4$ & 0.00825 & 0.02395 & -0.00502 & 0.00550 & 1.09639 & 0.00048 \\
\hline H46 - Au5 & 0.00854 & 0.02575 & -0.00416 & 0.00530 & 1.27292 & 0.00113 \\
\hline $\mathrm{C} 24-\mathrm{Au} 1$ & 0.01088 & 0.04032 & -0.00696 & 0.00852 & 1.22407 & 0.00156 \\
\hline $\mathrm{O} 28-\mathrm{Au} 3$ & 0.00386 & 0.01149 & -0.00177 & 0.00232 & 1.30929 & 0.00054 \\
\hline O29 - Au6 & 0.03139 & 0.12372 & -0.03478 & 0.03285 & 0.94460 & -0.00192 \\
\hline N50 - Au1 & 0.00689 & 0.01815 & -0.00379 & 0.00416 & 1.09833 & 0.00037 \\
\hline $\mathrm{O} 55$ - Au2 & 0.02696 & 0.10319 & -0.02838 & 0.02709 & 0.95447 & -0.00129 \\
\hline N59 - Au2 & 0.00646 & 0.01718 & -0.00343 & 0.00386 & 1.12580 & 0.00043 \\
\hline $\mathrm{O} 62-\mathrm{Au} 1$ & 0.01209 & 0.04199 & -0.00883 & 0.00966 & 1.09391 & 0.00083 \\
\hline H73 - Au3 & 0.00968 & 0.02810 & -0.00504 & 0.00603 & 1.19674 & 0.00099 \\
\hline $\mathrm{O} 65-\mathrm{Au} 2$ & 0.03707 & 0.15481 & -0.04359 & 0.04115 & 0.94389 & -0.00244 \\
\hline $\mathrm{H} 73$ - Au2 & 0.01030 & 0.03341 & -0.00587 & 0.00711 & 1.21065 & 0.00123 \\
\hline \multicolumn{7}{|c|}{ Medium Au-A interactions (CPGN/FU1) } \\
\hline O29 - Au6 & 0.02995 & 0.11675 & -0.03266 & 0.03092 & 0.94676 & -0.00173 \\
\hline $\mathrm{O} 55-\mathrm{Au} 2$ & 0.02901 & 0.11205 & -0.03126 & 0.02964 & 0.94796 & -0.00162 \\
\hline \multicolumn{7}{|c|}{ Medium Au-A interactions (CPGN/FU3) } \\
\hline O29-Au6 & 0.02710 & 0.10324 & -0.02844 & 0.02712 & 0.95379 & -0.00131 \\
\hline $\mathrm{O} 25-\mathrm{Au} 1$ & 0.01758 & 0.06279 & -0.01570 & 0.01571 & 0.99974 & -0.00001 \\
\hline
\end{tabular}




\begin{tabular}{|c|c|c|c|c|c|c|}
\hline O55 - Au2 & 0.03180 & 0.12638 & -0.03538 & 0.03349 & 0.94642 & -0.00189 \\
\hline $\mathrm{O} 65-\mathrm{Au} 2$ & 0.03061 & 0.11855 & -0.03336 & 0.03150 & 0.94416 & -0.00186 \\
\hline $\mathrm{O} 28-\mathrm{H} 72$ & 0.02050 & 0.06254 & -0.01615 & 0.01589 & 0.98390 & -0.00026 \\
\hline O64 - H31 & 0.02882 & 0.08955 & -0.02293 & 0.02266 & 0.98805 & -0.00027 \\
\hline \multicolumn{7}{|c|}{ Medium Au-A interactions (CPGN/FU4) } \\
\hline O29 - Au6 & 0.03087 & 0.12110 & -0.03399 & 0.03213 & 0.94528 & -0.00186 \\
\hline $\mathrm{O} 55-\mathrm{Au} 2$ & 0.02239 & 0.08028 & -0.02205 & 0.02106 & 0.95493 & -0.00099 \\
\hline $\mathrm{H} 51-\mathrm{O} 64$ & 0.01568 & 0.04680 & -0.01196 & 0.01183 & 0.98896 & -0.00013 \\
\hline H31 - O64 & 0.01637 & 0.05301 & -0.01331 & 0.01328 & 0.99759 & -0.00003 \\
\hline \multicolumn{7}{|c|}{ Medium Au-A interactions (CPGN/FU5) } \\
\hline O29 - Au6 & 0.03135 & 0.12342 & -0.03472 & 0.03278 & 0.94435 & -0.00193 \\
\hline $\mathrm{O} 64-\mathrm{Au} 2$ & 0.04041 & 0.17285 & -0.04867 & 0.04594 & 0.94389 & -0.00273 \\
\hline \multicolumn{7}{|c|}{ Medium Au-A interactions (CPGN/FU6) } \\
\hline O29 - Au6 & 0.03336 & 0.13357 & -0.03771 & 0.03555 & 0.94272 & -0.00216 \\
\hline \multirow[t]{2}{*}{ O65 - Au2 } & 0.03794 & 0.15756 & -0.04471 & 0.04205 & 0.94048 & -0.00266 \\
\hline & & & $\mathrm{GN} / \mathrm{H}_{2} \mathrm{O}$ & & & \\
\hline O7-Au5 & 0.04766 & 0.21010 & -0.06032 & 0.05642 & 0.93535 & -0.00390 \\
\hline
\end{tabular}

The values of $\rho(r), \nabla^{2} \rho, H_{b}, G_{b}, V_{b}$ and $-G_{b} / V_{b}$ along with Au-A interactions $\left(H_{b}<0\right)$ and medium hydrogen bonds in other structures were investigated, as shown in Table 3. CPGN/FU1 is the second stable configuration which has 2 medium $\mathrm{Au}-\mathrm{A}$ interactions and 19 weak interactions with $\rho_{a v}=0.0106$ and $\nabla^{2} \rho_{a v}=0.0355$ and it consists of 2 weak hydrogen bonds. Concerning the structure of CPGN/FU2, the strength and nature of Au-A interactions have higher values and this has made this structure more stable than CPGN/FU1.

CPGN/FU6 is the third stable configuration. In this structure, there are $17 \mathrm{Au}-\mathrm{A}$ interactions with 2 medium interactions and 15 weak interactions of $\mathrm{Au}-\mathrm{A}$ with $\rho_{a v}=0.0118$ and $\nabla^{2} \rho_{a v}=0.0412$. CPGN/FU6 has 2 weak hydrogen bonds. The order of stability of the other configurations is as follows (Table 3).

CPGN/FU4 has 2 medium Au-A interactions and 16 weak interactions with values of $\rho_{a v}=0.0111$ and $\nabla^{2} \rho_{a v}=$ 0.0372. Also, this structure has 2 hydrogen bonds of H51 ‥ O64 and H31 ‥ O64 as a medium hydrogen bond and one weak hydrogen bond. CPGN/FU3 has 4 medium Au-A interactions and 15 weak interactions with $\rho_{a v}=0.0120$ and $\nabla^{2} \rho_{a v}=0.0409$ and 2 medium hydrogen bonds of $\mathrm{H} 31 \cdots \mathrm{O} 64$ and $\mathrm{H} 72 \cdots \mathrm{O} 28$ and one weak hydrogen bond. CPGN/FU5 has 2 medium interactions of Au-A and 16 weak interactions with the values of $\rho_{a v}=0.0117$ and $\nabla^{2} \rho_{a v}=0.0415$. In this structure, there is 1 weak hydrogen bond.

As previously mentioned, we used PCM to consider the implicit effect of the solvent. QTAIM calculations can be used to justify that there is no need to consider the explicit effect of the solvent. To do this, we must show that there is no chemical bond between $\mathrm{GN}$ and $\mathrm{H}_{2} \mathrm{O}$ molecules (solvent). The $\mathrm{GN}$ structure was optimized next to $\mathrm{H}_{2} \mathrm{O}\left(\mathrm{GN} / \mathrm{H}_{2} \mathrm{O}\right)$ and then AIM calculations were performed. The optimized structure of $\mathrm{GN} / \mathrm{H}_{2} \mathrm{O}$ along with the molecular graph and the values of $\rho(r), \nabla^{2} \rho, H_{b}, G_{b}, V_{b}$ and $-G_{b} / V_{b}$ are represented in Fig. 4 and Table 3 , respectively. According to the bond length in Fig. 4 (Au-O) and the values in Table 3, no chemical bond is formed between GN and $\mathrm{H}_{2} \mathrm{O}$.

\section{Conclusion}

In this study, seven gold nanoparticle-cyclic peptide configurations with the anticancer drug FU were investigated in the aqueous solution and gas phase at the M06-2X level. The values of binding energy indicate that the performance of CPGN with FU drug in CPGN/FU1-7 configurations is appropriate and the simultaneous interaction of the drug with $\mathrm{CP}$ and GN results in greater stability. The data obtained from solvation and binding energies show that the solubility of FU and GN increases in the presence of CP. The global hardness $(\eta)$ and energy gap $\left(E_{g}\right)$ for FU decrease in the presence of GN and CP (CPGN/FU1-7), indicating the reactivity FU increases. According to AIM studies, FU can interact with $\mathrm{CPGN}$ as $\mathrm{Au}-\mathrm{A}(\mathrm{A}=\mathrm{N}, \mathrm{O}, \mathrm{F}, \mathrm{C}, \mathrm{H})$ and hydrogen bonding. Most Au-A interactions with $-G_{b} / V_{b}>1$, $H_{b}>0$ and $\nabla^{2} \rho>0$ are weak interactions. AIM results showed that in the most unstable configuration (CPGN/FU7), 

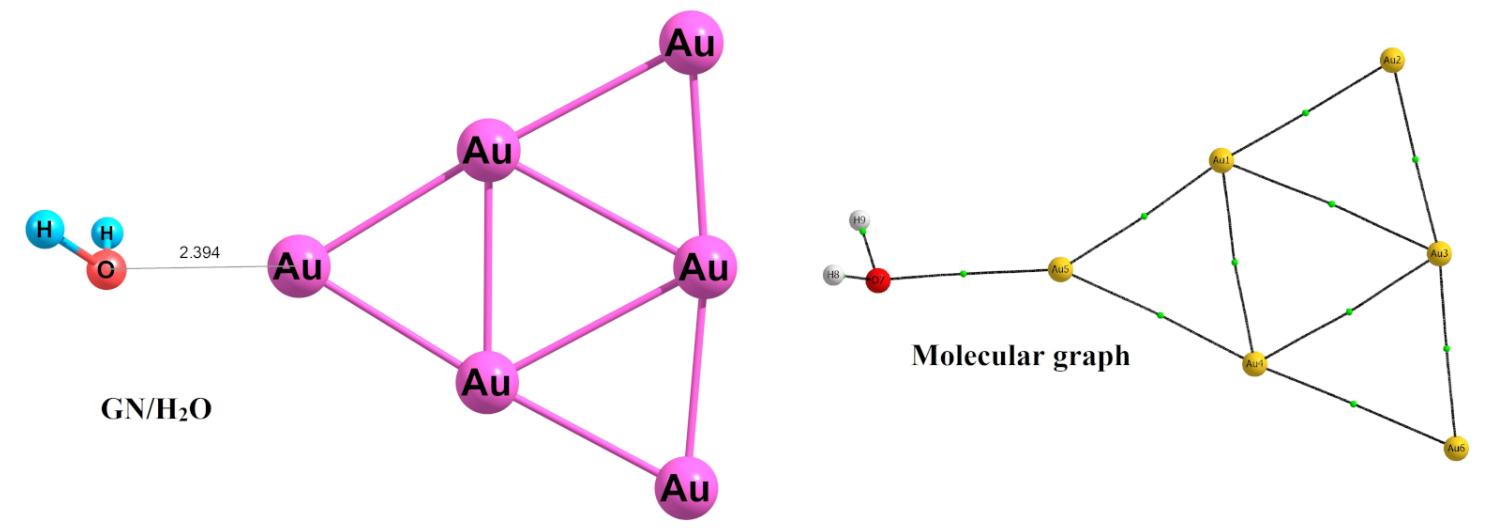

FIG. 4. Optimized structure and molecular graph of $\mathrm{GN} / \mathrm{H}_{2} \mathrm{O}$. Small green spheres and lines related to the bond critical points $(\mathrm{BCP})$ and the bond paths, respectively

the drug interacts only with GN, and in the most stable configuration (CPGN/FU2), Au-A interactions are stronger and are accompanied by hydrogen bonds.

\section{Acknowledgements}

We thank the Research Centre for Animal Development Applied Biology for allocation of computer time.

\section{References}

[1] Girase M.L., Patil P.G., Ige P.P., Biomaterials P. Polymer-drug conjugates as nanomedicine: a review. Int. J. Polymer. Mater., 2020, 69, P. 990-1014.

[2] Rahbar M., Morsali A., Bozorgmehr M.R., Beyrmabadi S.A. Quantum chemical studies of chitosan nanoparticles as effective drug delivery systems for 5-fluorouracil anticancer drug. J. Mol. Liq., 2020, 302, 112495.

[3] Lotfi M., Morsali A., Bozorgmehr M.R. Comprehensive quantum chemical insight into the mechanistic understanding of the surface functionalization of carbon nanotube as a nanocarrier with cladribine anticancer drug. Appl. Surf. Sci., 2018, 462, P. 720-729.

[4] Samal S.K. Cancer and Carbon Nano Tubes: A Promising Hope of Future. Scientific Review, 2018, 4, P. 64-67.

[5] Connor D.M., Broome A.-M. Gold Nanoparticles for the Delivery of Cancer Therapeutics. Adv. Cancer Res., 2018,139, P. $163-184$.

[6] Roohi R., Emdad H., Jafarpur K. A comprehensive study and optimization of magnetic nanoparticle drug delivery to cancerous tissues via external magnetic field. J. Test. Eval., 2019, 47, P. 681-703.

[7] Shabani Z., Morsali A., Bozorgmehr M.R., Beyramabadi S.A. Quantum chemical modeling of iron oxide magnetic nanoparticles functionalized with cytarabine. Chem. Phys. Lett., 2019, 719, P. 12-21.

[8] Foglia L.M., Alvarez S.G., et al. Recent patents on the synthesis and application of silica nanoparticles for drug delivery. Recent Pat. Biotechnol., 2011, 5, P. 54-61.

[9] Singh R.K., Kim H.W. Inorganic nanobiomaterial drug carriers for medicine. Tissue Eng. Regen. Med., 2013, 10, P. 296-309.

[10] Ostroushko A.A., Gagarin I.y.D., Danilova I.G., Gette I.F. The use of nanocluster polyoxometalates in the bioactive substance delivery systems. Nanosystems: Phys. Chem. Math., 2019, 10, P. 318-349.

[11] Popova N., Popov A., Shcherbakov A., Ivanov V. Layer-by-layer capsules as smart delivery systems of $\mathrm{CeO}_{2}$ nanoparticle-based theranostic agents. Nanosystems: Phys. Chem. Math., 2017, 8, P. 282-289.

[12] Fan M., Han Y., et al. Ultrasmall gold nanoparticles in cancer diagnosis and therapy. Theranostics, $2020,10,4944$.

[13] Haume K., Rosa S., et al. Gold nanoparticles for cancer radiotherapy: a review. Cancer Nanotechnol., 2016,7 , P. 8-28.

[14] Xu Y., He R., et al. Laser beam controlled drug release from Ce6-gold nanorod composites in living cells: a FLIM study. Nanoscale, 2015, 7, P. 2433-2441.

[15] Khutale G.V., Casey A. Synthesis and characterization of a multifunctional gold-doxorubicin nanoparticle system for pH triggered intracellular anticancer drug release. Eur. J. Pharm. Biopharm., 2017, 119, P. 372-380.

[16] Fernandez-Lopez S., Kim H.-S., et al. Antibacterial agents based on the cyclic D, L- $\alpha$-peptide architecture. Nature, $2001,412,452$.

[17] Larnaudie S.C., Brendel J.C., et al. Cyclic Peptide-Polymer Nanotubes as Efficient and Highly Potent Drug Delivery Systems for Organometallic Anticancer Complexes. Biomacromolecules, 2018, 19, P. 239-247.

[18] Ellison A.J., Tanrikulu I.C., Dones J.M., Raines R.T. Cyclic Peptide Mimetic of Damaged Collagen. Biomacromolecules, 2020, 21, P. 1539_ 1547.

[19] Shahabi M., Raissi H. Assessment of solvent effects on the inclusion behavior of pyrazinamide drug into cyclic peptide based nanotubes as novel drug delivery vehicles. J. Mol. Liq., 2018, 268, P. 326-334.

[20] Nasrolahi Shirazi A., Tiwari R.K., et al. Cyclic peptide-selenium nanoparticles as drug transporters. Mol. Pharm., 2014, 11, P. $3631-3641$.

[21] Chermahini A.N., Farrokhpour H., Shahangi F., Dabbagh H.A. Cyclic peptide nanocapsule as ion carrier for halides: a theoretical survey. Struct. Chem., 2018, 29, P. 1351-1357.

[22] Dartois V., Sanchez-Quesada J., et al. Systemic antibacterial activity of novel synthetic cyclic peptides. Antimicrob. Agents Chemother., 2005, 49, P. 3302-3310.

[23] Khalfa A., Tarek M. On the antibacterial action of cyclic peptides: insights from coarse-grained MD simulations. J. Phys. Chem. B, 2010, 114, P. 2676-2684. 
[24] Claro B., Bastos M., Garcia-Fandino R. Design and applications of cyclic peptides. In Peptide Applications in Biomedicine, Biotechnology and Bioengineering, 2018, Elsevier, P. 87-129.

[25] Zorzi A., Deyle K., Heinis C. Cyclic peptide therapeutics: past, present and future. Curr. Opin. Chem. Biol., 2017,38 , P. $24-29$.

[26] Park S.E., Sajid M.I., Parang K., Tiwari R.K.M.p. Cyclic Cell-Penetrating Peptides as Efficient Intracellular Drug Delivery Tools. Mol. Pharm., 2019, 16, P. 3727-3743.

[27] Oh D., Nasrolahi Shirazi A., et al. Enhanced cellular uptake of short polyarginine peptides through fatty acylation and cyclization. Mol. Pharm., 2014, 11, P. 2845-2854.

[28] Mandal D., Nasrolahi Shirazi A., Parang K. Cell-penetrating homochiral cyclic peptides as nuclear-targeting molecular transporters. Angew. Chem. Int. Ed., 2011, 50, P. 9633-9637.

[29] Jing X., Jin K.J.M.R.R. A gold mine for drug discovery: Strategies to develop cyclic peptides into therapies. Med. Res. Rev., 2020, 40, P. 753-810.

[30] Dougherty P.G., Sahni A., Pei D. Understanding cell penetration of cyclic peptides. Chem. Rev., 2019, 119, P. $10241-10287$.

[31] Nasrolahi Shirazi A., Tiwari R.K., et al. Surface decorated gold nanoparticles by linear and cyclic peptides as molecular transporters. Mol. Pharm., 2013, 10, P. 3137-3151.

[32] Naghavi F., Morsali A., Bozorgmehr M.R., Beyramabadi S.A. Quantum molecular study of mesoporous silica nanoparticle as a delivery system for troxacitabine anticancer drug. J. Mol. Liq., 2020, 310, 113155.

[33] Nasrabadi M., Morsali A., Beyramabadi S.A. An applied quantum-chemical model for genipin-crosslinked chitosan (GCS) nanocarrier. Int. J. Biol. Macromol., 2020, 165, P. 1229-1240.

[34] Bokarev A., Plastun I. Possibility of drug delivery due to hydrogen bonds formation in nanodiamonds and doxorubicin: molecular modeling. Nanosystems: Phys. Chem. Math., 2018, 9, P. 370-377.

[35] Nasrabadi M., Beyramabadi S.A., Morsali A. Surface functionalization of chitosan with 5-nitroisatin. Int. J. Biol. Macromol., 2020, 147, P. 534-536.

[36] Naghavi F., Morsali A., Bozorgmehr M.R. Molecular mechanism study of surface functionalization of silica nanoparticle as an anticancer drug nanocarrier in aqueous solution. J. Mol. Liq., 2019, 282, P. 392-400.

[37] Hadi L., Ali M., Momen H.M. The prediction of COOH functionalized carbon nanotube application in melphalan drug delivery. Nanosystems: Phys. Chem. Math., 2019, 10, P. 438-446.

[38] Sara J.D., Kaur J., et al. 5-fluorouracil and cardiotoxicity: a review. Ther. Adv. Med. Oncol., 2018, 10, 1758835918780140.

[39] Longley D.B., Harkin D.P., Johnston P.G. 5-fluorouracil: mechanisms of action and clinical strategies. Nat. Rev. Cancer, 2003, 3, P. 330-338.

[40] Metterle L., Nelson C., Patel N. Intralesional 5-fluorouracil (FU) as a treatment for nonmelanoma skin cancer (NMSC): a review. J. Am. Acad. Dermatol., 2016, 74, P. 552-557.

[41] Lee J.J., Beumer J.H., Chu E. Therapeutic drug monitoring of 5-fluorouracil. Cancer Chemother. Pharmacol., 2016, 78, P. 447-464.

[42] Chen L., Yu H., et al. Fabrication of a microporous Dy (III)-organic framework with polar channels for 5-Fu (fluorouracil) delivery and inhibiting human brain tumor cells. Struct. Chem., 2018, 29, P. 1885-1891.

[43] Frisch M., Trucks G., et al. G09 Gaussian Inc. Gaussian 09, revision B.01. Gaussian, Inc., Wallingford, CT, 2009.

[44] Parr R.G., Szentpaly L.v., Liu S. Electrophilicity index. J. Am. Chem. Soc., 1999, 121, P. $1922-1924$.

[45] AIMAll TK Gristmill Software. Overland Park KS, USA, 2016, URL: aim.tkgristmill.com.

[46] Poteau R., Trinquier G. All-cis cyclic peptides. J. Am. Chem. Soc., 2005, 127, P. 13875-13889.

[47] Manzoor D., Pal S., Krishnamurty S. Influence of charge and ligand on the finite temperature behavior of gold clusters: a BOMD study on Au6 cluster. J. Phys. Chem. C, 2013, 117, P. 20982-20990.

[48] Fagan J.W., Weerawardene K.D.M., et al. Toward quantitative electronic structure in small gold nanoclusters. J. Chem. Phys., 2021, 155, 014301.

[49] Shi H.X., Sun W.G., et al. Probing the Interactions of $\mathrm{O}_{2}$ with Small Gold Cluster $\mathrm{Au}_{n} \mathrm{Q}(n=2-10, \mathrm{Q}=0-1)$ : A Neutral Chemisorbed Complex $\mathrm{Au}_{5} \mathrm{O}_{2}$ Cluster Predicted. J. Phys. Chem. C, 2017, 121, P. 24886-24893.

[50] Ligare M.R., Morrison K.A., et al. Ion Mobility Spectrometry Characterization of the Intermediate Hydrogen-Containing Gold Cluster $\mathrm{Au}_{7}\left(\mathrm{PPh}_{3}\right)_{7} \mathrm{H}_{5}^{2+}$. J. Phys. Chem. Lett., 2021, 12, P. 2502-2508.

[51] Lang S.M., Bernhardt T.M., et al. Selective C-H bond cleavage in methane by small gold clusters. Angew. Chem. Int. Ed. Engl., 2017, 129, P. 13591-13595.

[52] Gilb S., Weis P., et al. Structures of small gold cluster cations $\left(\mathrm{Au}_{n}^{+}, n<14\right)$ : Ion mobility measurements versus density functional calculations. J. Chem. Phys., 2002, 116, P. 4094-4101.

[53] Lechtken A., Neiss C., Kappes M.M., Schooss D. Structure determination of gold clusters by trapped ion electron diffraction: Au $14-\mathrm{Au}_{19}^{-}$. Phys. Chem. Chem. Phys., 2009, 11, P. 4344-4350.

[54] Rozas I., Alkorta I., Elguero J. Behavior of ylides containing N, O, and C atoms as hydrogen bond acceptors. J. Am. Chem. Soc., 2000, 122, P. 11154-11161.

[55] Espinosa E., Souhassou M., Lachekar H., Lecomte C. Topological analysis of the electron density in hydrogen bonds. Acta Crystallogr. Sect. B: Struct. Sci., 1999, 55, P. 563-572.

[56] Espinosa E., Alkorta I., Elguero J., Molins E. From weak to strong interactions: A comprehensive analysis of the topological and energetic properties of the electron density distribution involving X-H F-Y systems. J. Chem. Phys., 2002, 117, P. 5529-5542. 